\title{
Design of Improved Algorithm and Model for Multi-constrained Fuzzy Predictive Analysis
}

\author{
Junhui Song ${ }^{1 *}$, Hua Xie ${ }^{2}$, Liping $\mathrm{Shi}^{1}$ \\ ${ }^{1}$ Xinyang Normal University, College of Computer and Information Technology, Xinyang, 464000, China \\ ${ }^{2}$ Xinyang Vocational and Technical College, President's Office, Xinyang, 464000, China
}

Corresponding Author Email: songyuan0520@126.com

https://doi.org/10.18280/ria.330610

Received: 25 May 2019

Accepted: 10 August 2019

\section{Keywords:}

fuzzy predictive analysis, multiple constraints, system engineering, fuzzy theory, algorithm and model

\begin{abstract}
Complex system engineering often has high fuzziness and multiple constraints. In this case, it is difficult to achieve consistent results through predictive analysis. To solve the problem, this paper explores the key techniques and methods for predictive analysis on complex systems, and puts forward an improved strategy for multi-constrained fuzzy predictive analysis. The author explained the normalization, weighting, granularity setting and classic domain of the attributes of multiple constraints, introduced the calculation of the fuzzy distance and fuzzy closeness for the attributes of multiple constraints, and detailed the realization of our algorithm and model multi-constrained fuzzy predictive analysis. The effectiveness and feasibility of our algorithm and model were demonstrated through comparison with relevant data in the literature. The results show that the results of our approach agree with those of the literature. The proposed algorithm and model provide a good theoretical basis to predictive analysis of complex system engineering, especially that with multiple fuzzy constraints.
\end{abstract}

\section{INTRODUCTION}

The problem with multiple constraints (multi-constrained problem) is a difficulty in system engineering analysis. In many fields of engineering, multi-constrained problem calls for optimal design and analysis, especially for complex system engineering $[1,2]$. To make matters worse, the presence of multi-constrained problem adds to the unpredictability in the development of complex systems. Thus, it is very difficult to predict the trend of complex systems in a scientific, accurate and reliable manner $[3,4]$. To overcome the difficulty, the key lies in capturing the constraints, especially when they are fuzzy and uncertain, accurately, and using them to make effective predictions of the trend of complex systems. Doing so could provide powerful support to the design, maintenance and repair of complex systems, making them more applicable in engineering cases.

Currently, fruitful results have been achieved on the prediction and analysis of fuzzy systems. Some scholars predicted the trend of fuzzy systems based on the gray theory. $\mathrm{Lu}$ [5] discussed the multi-attribute prediction of complex systems based on grey relational analysis (GRA), and presented a grey prediction model for the implicit associations in complex systems. Through the GRA, Javed et al. [6] optimized the shape and analyzed the thermal properties of heat exchangers, providing an important reference for industrial thermal analysis and gray multi-objective optimization. Based on the gray model, Bezuglov and Comert [7] predicted the traffic parameters (e.g. speed, travel time and flow) in smart transport system in an accurate manner. Memon et al. [8] combined the gray system theory with the uncertainty theory to study the supplier selection problem with random uncertainty and identifiable uncertainty.

Some scholars predicted the trend of fuzzy systems based on neural networks (NNs). Vukovic et al. [9] analyzed a
Sharpe ratio prediction NN model, and proved that the NN can successfully forecast the nonlinear time lag sequences. Navas et al. [10] applied different artificial neural networks (ANNs) and empirical classification regression models to predict wind speed, and estimated the wind speed using the SPSS software. Using the growing and pruning method, Akyol [11] determined the ideal parameters (i.e. the optimal number of hidden layers, the optimal number of hidden layer neurons, and the activation function) of deep NNs and extreme learning machines, and verified the prediction ability of the designed model. Liu et al. [12] implemented the uncertain genetic neural network in the prediction of landslide risk, and achieved good engineering application effect.

Some scholars predicted the trend of fuzzy systems based on support vector machine (SVM). Wadkar et al. [13] adopted SVM-based technique to detect malwares. Hou et al. [14] developed a projection nonparallel SVM for pattern classification, and applied it to recognize and analyze the patterns of complex systems. Lukmanto et al. [15] integrated feature selection and fuzzy SVM for early detection and prediction of diabetes mellitus. Gangsar et al. [16] studied the fault diagnosis and prediction of induction motors based on the SVM.

Some scholars predicted the trend of fuzzy systems based on the genetic algorithm (GA), and applied the results in the combinatory optimization and decision-making of complex systems. Lee [17] reviewed the applications of the GA in operations management. Iyer et al. [18] applied the GA in the design and economic optimization of shell-and-tube heat exchanger. de Assis. et al. [19] identified Volterra systems with the aid of the GA. Tseng et al. [20] investigated disassembly sequence planning based on the GA.

Some scholars predicted the trend of fuzzy systems based on the extension theory. $\mathrm{Lu}$ [21] designed a multilevel extension association forecast model under the influence of 
multiple factors. Chao [22] combined the extension theory and traditional perturbation and observation method, and relied on the combined approach to design a maximum power tracker based on the particle swarm optimization (PSO). Drawing on the extension theory, Chao et al. [23] analyzed the maximum power point (MPP) tracking of photovoltaic (PV) systems.

To sum up, the above research results have been applied successfully in engineering. However, there is ample room for improvement in the following aspects due to the unknown nature of fuzzy information: the effective measurement of fuzzy information, and more refined and accurate prediction of information. Therefore, this paper carries out an in-depth analysis on multi-constrained fuzzy prediction of complex system engineering based on the fuzzy system theory [24-26]. The key contents include normalizing multi-constrained fuzzy information, fuzziness calculation, and the steps of fuzzy prediction. On this basis, the author developed an algorithm and model for improved multi-constrained fuzzy prediction.

The remainder of this paper is organized as follows: Section 2 introduces the basic concepts of multi-constrained fuzzy predictive analysis; Section 3 presents our algorithm and model for multi-constrained fuzzy prediction, explains the normalization, weighting, granularity setting and classic domain of the attributes of multiple constraints, and details the steps of multi-constrained fuzzy predictive analysis; Section 4 verified our algorithm and model for multi-constrained fuzzy prediction through comparative analysis; Section 5 puts forward the conclusions of this research.

\section{BASIC CONCEPTS}

\subsection{Attribute set of multi-constrained fuzzy predictive analysis}

The term multi-constrained has multiple meanings. First, the design goals for the design, analysis and optimization of complex systems are constrained by various factors. To achieve ideal design results, the relationship between design constraints and design goals must be handled well. Second, the design requirements and design goals for complex systems might be contradictory or incompatible. The relationship between the relevant influencing factors must be sorted out to solve the contradiction or incompatibility. Third, there are implicit or explicit correlations between the design attributes of complex systems, which may affect the system performance. To improve the design and predictive analysis of multiconstrained fuzzy systems, this paper attempts to extract the features and attributes of the multiple constraints, forming the attribute set of multi-constrained fuzzy predictive analysis. Let $Q$ be the number of the extracted attributes of the multiple constraints and $S$ be each extracted attribute. Then, the attribute set $\boldsymbol{S}$ of multi-constrained fuzzy predictive analysis, a.k.a. the fuzzy set, can be expressed as:

$$
\boldsymbol{S}=\left\{S_{1}, S_{2}, \cdots, S_{k}, \cdots, S_{Q-1}, S_{Q}\right\}
$$

\subsection{Fuzzy distance}

In fuzzy system theory, the fuzzy distance is defined to reflect the closeness between the predictive analysis target and the fuzzy set $\boldsymbol{S}$. Let $v_{k}=\left[v_{k}(a), v_{k}(b)\right]$ be the fuzziness of the attribute $k$ corresponding to the predictive analysis target, and $v_{k}^{S}=\left[v_{k}^{S}(a), v_{k}^{S}(b)\right]$ be the fuzziness interval of attribute $k$. Then, the fuzzy distance between the target and the attribute can be defined as:

$$
D_{k}=\sqrt[P]{\left(\left|v_{k}^{S}(a)-v_{k}(a)\right|^{P}+\left|v_{k}^{S}(b)-v_{k}(b)\right|^{P}\right) / 2}
$$

If $P=1$, then:

$$
D_{k}=\left(\left|v_{k}^{S}(a)-v_{k}(a)\right|+\left|v_{k}^{S}(b)-v_{k}(b)\right|\right) / 2
$$

In this case, the distance is the Hamming distance.

If $P=2$, then:

$$
D_{k}=\sqrt{\left(\left|v_{k}^{S}(a)-v_{k}(a)\right|^{2}+\left|v_{k}^{S}(b)-v_{k}(b)\right|^{2}\right) / 2}
$$

In this case, the distance is the Euclidean distance.

\section{ALGORITHM AND MODEL OF MULTI- CONSTRAINED FUZZY PREDICTIVE ANALYSIS}

\subsection{Normalization of the attributes of multiple constraints}

In predictive analysis, different indices may differ in dimensions. To make the analysis results more reliable, all the indices should have uniform metrics. Since the predictive analysis in this research is fuzzy, it is assumed that the value of each attribute in predictive analysis falls into a fuzzy interval, i.e. the initial value of index $k$ corresponding to the predictive analysis target is $v_{k}=\left[v_{k}(a), v_{k}(b)\right]$. Let $u_{k}=$ $\left[u_{k}(a), u_{k}(b)\right]$ be the normalized correlation, and $v_{k}^{\Theta}=$ $\left[v_{k}^{\Theta}(a), v_{k}^{\Theta}(b)\right]$ be the threshold of index $k$. Then, the normalization of the cost-based index $k$ can be described as:

$$
\left\{\begin{array}{l}
u_{k}=\left[u_{k}(a), u_{k}(b)\right] \\
u_{k}(a)=\frac{v_{k}^{\Theta}(b)-v_{k}(b)}{v_{k}^{\Theta}(b)-v_{k}^{\Theta}(a)} \\
u_{k}(b)=\frac{v_{k}^{\Theta}(b)-v_{k}(a)}{v_{k}^{\Theta}(b)-v_{k}^{\Theta}(a)}
\end{array}\right.
$$

The normalization of the benefit-based index $k$ can be described as:

$$
\left\{\begin{array}{l}
u_{k}=\left[u_{k}(a), u_{k}(b)\right] \\
u_{k}(a)==\frac{v_{k}(a)-v_{k}^{\Theta}(a)}{v_{k}^{\Theta}(b)-v_{k}^{\Theta}(a)} \\
u_{k}(b)=\frac{v_{k}(b)-v_{k}^{\Theta}(a)}{v_{k}^{\Theta}(b)-v_{k}^{\Theta}(a)}
\end{array}\right.
$$




\subsection{Weighing of the attributes of multiple constraints}

The weights of the attributes of multiple constrains can be determined by various methods, such as the analytic hierarchy process (AHP), entropy weight method, comprehensive evaluation, the $\mathrm{NN}$ method and gray correlation method. Considering its simplicity, practicality and result reliability, the AHP was adopted for the weighting of attributes of multiple constraints.

Step 1. Create the attribute set of multi-constrained fuzzy predictive analysis.

Step 2. Perform expert scoring based on a nine-point scale, producing the judgement matrix A:

$$
\mathbf{A}=\left\{a_{i j}\right\}_{n x n}=\left[\begin{array}{cccc}
a_{11} & a_{12} & \cdots & \cdots \\
a_{21} & a_{22} & \cdots & \cdots \\
\cdots & \cdots & \cdots & \cdots \\
\cdots & \cdots & \cdots & a_{n n}
\end{array}\right]
$$

Step 3. Normalize the judgement matrix A:

$$
\bar{a}_{j}=\sum_{i=1}^{n}\left(a_{i j} / \sum_{i=1}^{n} a_{i j}\right)
$$

Step 4. Obtain the maximum characteristic root of judgement matrix A:

$$
\lambda_{\max }=\sum_{j=1}^{n} \frac{(A W)_{i}}{n W_{i}}
$$

Step 5. Check the consistency of judgement matrix A:

$$
\left\{\begin{array}{l}
C I=\frac{\lambda_{\max }-n}{n-1} \\
C R=C I / R I
\end{array}\right.
$$

If $C R \leq 0.1$, then judgement matrix $\mathbf{A}$ has good consistency, and proceed with the next step; Otherwise, reestablish the judgement matrix.

Step 6. Acquire the weights:

$$
w_{j}=\bar{a}_{j} / \sum_{j=1}^{n} \bar{a}_{j}
$$

\subsection{Granularity setting for the attributes of multiple constraints}

The granularity setting for the attributes of multiple constraints refers to the division of the value interval of the attribute set for multi-constrained fuzzy predictive analysis. The granularity should be properly configured according to the actual condition of the predictive analysis target. If the granularity is too small, the attribute set will be divided into too many items. In extreme cases, each item is a reference object, making it impossible to predict the target. If the granularity is too large, the attribute set will be divided too coarsely. In this case, the range of the reference object is too broad, so is that of the prediction result. Then, it is also impossible to accurately predict the target.

\subsection{Classic fields for the attributes of multiple constraints}

Based on the granularity set in the preceding subsection, the attributes of multiple constraints can be divided into $\mathrm{H}$ classic fields. Then, the interval of the reference object $h$ in each classic field of the attribute set can be expressed as $Z_{h}=$ $\left[Z_{h}(a), Z_{h}(b)\right]$. The size of this interval determines the reference objects in the corresponding historical records. Suppose there are $\mathrm{N}$ reference objects. Then, the classic field $\Omega_{k}^{h}$ of the reference object $h$ formed by these reference object relative to index $k$ can be expressed as:

$$
\left\{\begin{array}{l}
\Omega_{k}^{h}=\left[\Omega_{k}^{h}(a), \Omega_{k}^{h}(b)\right] \\
\Omega_{k}^{h}(a) \leq \min _{1 \leq i \leq N}\left(u_{k}=\left[u_{k}^{i}(a), u_{k}^{i}(b)\right]\right) \\
\Omega_{k}^{h}(b) \geq \max _{1 \leq i \leq N}\left(u_{k}=\left[u_{k}^{i}(a), u_{k}^{i}(b)\right]\right)
\end{array}\right.
$$

To ensure the reliability and accuracy of prediction results, the following values are selected to make the predicted range wider:

$$
\left\{\begin{array}{l}
\Omega_{k}^{h}(a)<\min _{1 \leq i \leq N} u_{k}^{i}(a) \\
\Omega_{k}^{h}(b)>\max _{1 \leq i \leq N} u_{k}^{i}(b)
\end{array}\right.
$$

\subsection{Fuzzy closeness for the attributes of multiple constraints}

Let $u_{k}=\left[u_{k}(a), u_{k}(b)\right]$ be the value of predictive analysis targets relative to index $k$. Then, the fuzzy distance $D_{k}^{h}$ between $u_{k}=\left[u_{k}(a), u_{k}(b)\right]$ and the reference object $h$ can be computed by:

$$
D_{k}^{h}=\sqrt[P]{\left(\left|\Omega_{k}^{h}(a)-u_{k}(a)\right|^{P}+\left|\Omega_{k}^{h}(b)-u_{k}(b)\right|^{P}\right) / 2}
$$

In particular, if the value of the predictive analysis target relative to index $k$ is an exact value, i.e. $u_{k}=u_{k}(a)=u_{k}(b)$, then:

$$
D_{k}^{h}=\sqrt[P]{\left(\left|\Omega_{k}^{h}(a)-u_{k}\right|^{P}+\left|\Omega_{k}^{h}(b)-u_{k}\right|^{P}\right) / 2}
$$

The fuzzy distance $D_{k}^{h}$ is negatively correlated with the closeness between the two factors. Hence, the fuzzy closeness $\Phi_{k}^{h}$ can be introduced:

$$
\Phi_{k}^{h}=1-D_{k}^{h}
$$

Considering the weight of each attribute of the multiple constraints, the integrated weighted fuzzy closeness $\Phi_{k}^{h}$ can be described as:

$$
\Phi^{h}=\sum_{k=1}^{K}\left(w_{k} *\left(1-D_{k}^{h}\right)\right)
$$

The integrated weighted fuzzy closeness $\Phi_{k}^{h}$ has a negative 
correlation with the closeness between the predictive analysis target and the reference object.

\subsection{Realization of multi-constrained fuzzy predictive analysis}

Based on the integrated weighted fuzzy closeness $\Phi_{k}^{h}$, the classic field of the reference object $h$ corresponding to the predictive analysis target can be obtained as $Z_{h}=$
$\left[Z_{h}(a), Z_{h}(b)\right]$. Then, the fuzzy prediction $Z^{*}$ of the predictive analysis target must satisfy:

$$
\mathrm{Z}^{*} \in \mathrm{Z}_{h}=\left[\mathrm{Z}_{h}(a), \mathrm{Z}_{h}(b)\right]
$$

Therefore, the algorithm of multi-constrained fuzzy predictive analysis can be established. The workflow of the established algorithm is shown in Figure 1.

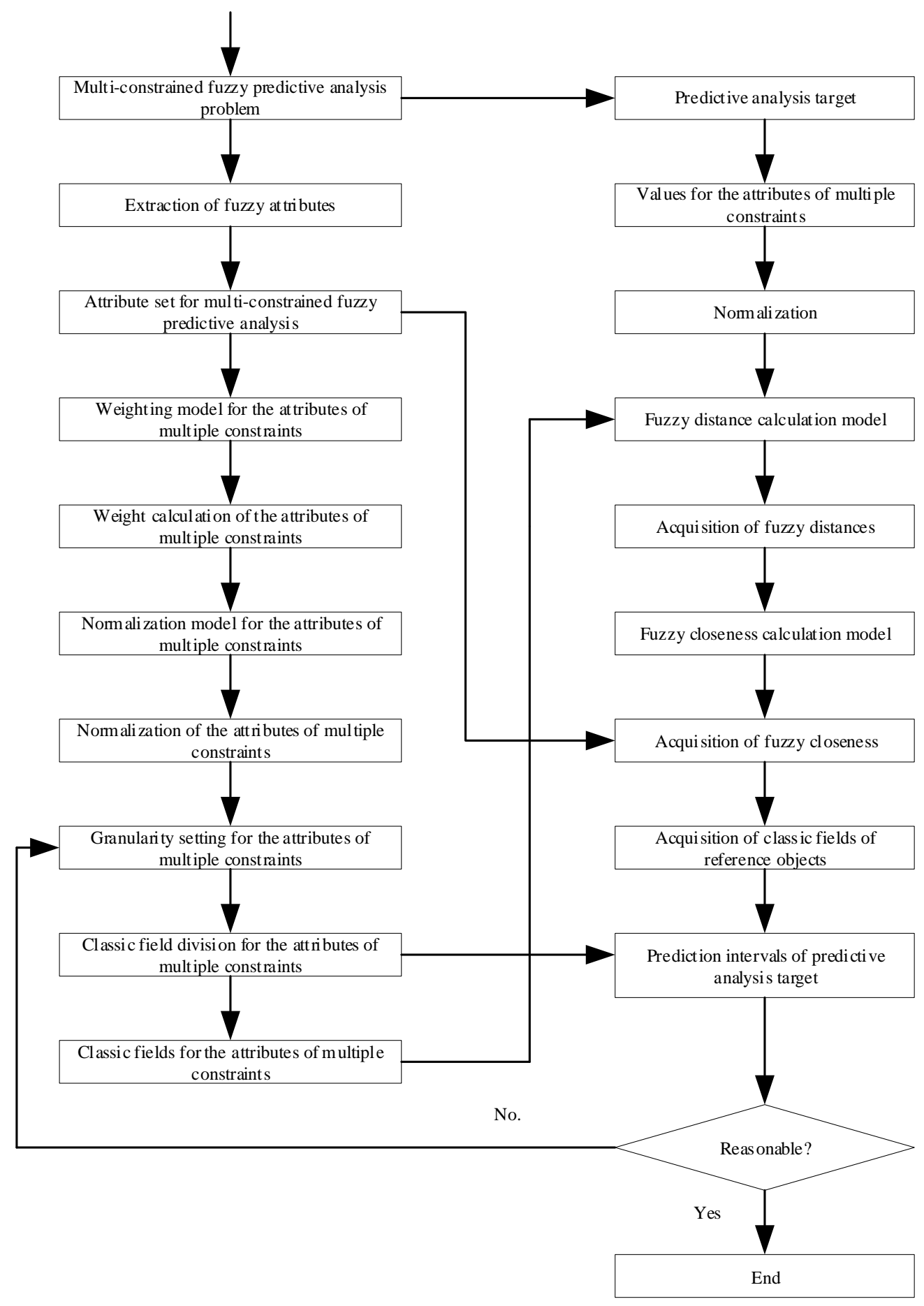

Figure 1. Workflow of multi-constrained fuzzy predictive analysis algorithm

\section{COMPARATIVE ANALYSIS}

This section aims to verify the feasibility and effectiveness of the proposed algorithm and model for multi-constrained fuzzy predictive analysis, and explain the realization of our algorithm and model. As mentioned before, the fuzzy distance is measured by Euclidean distance. The predictive analysis results of our algorithm and model were compared with the 
data in the research of $\mathrm{Lu}$ [5]. The initial data are compared in Table 1 below.

The fuzzy distance and fuzzy closeness were computed by the corresponding formulas in our algorithm, and compared with those in Lu Hui's research (Tables 2 and 3).

As shown in the data below, the prediction by our algorithm should fall into the target interval for 0.937 , i.e. $0.230-0.300$. The result agrees with the data in Lu Hui's research. However, the predictive analysis target was closer to target interval 1 for attribute 1, and closer to target interval 2 for attribute 2 than that in Lu Hui's research. There is no significant difference in this respective for attribute 3 . Since attribute 2 has the greatest weight, the final results were not obviously different.

Similarly, the prediction by our algorithm was compared with that of Kemal Akyol's research [11] (Tables 4-6).

The fuzzy distance and fuzzy closeness were computed by the corresponding formulas in our algorithm, and compared with those in Kemal Akyol's research (Tables 5 and 6).

It can be seen from Tables 5 and 6 that prediction by our algorithm should fall into the target interval for 0.939 , i.e. $0.180-0.280$. The result is consistent with the data in Kemal Akyol's research.

Table 1. The initial data of our algorithm and Lu Hui's research

\begin{tabular}{|c|c|c|c|c|}
\hline & \multicolumn{3}{|c|}{ Classic field } & \multirow{2}{*}{ Target interval } \\
\hline & Attribute 1 & Attribute 2 & Attribute 3 & \\
\hline 1 & $0-0.070$ & $0.135-0.195$ & $0.120-0.180$ & $0.070-0.150$ \\
\hline 2 & $0.070-0.180$ & $0.080-0.270$ & $0.110-0.200$ & $0.150-0.230$ \\
\hline 3 & $0.130-0.340$ & $0.200-0.260$ & $0.340-0.400$ & $0.230-0.300$ \\
\hline Predictive analysis target & 0.247 & 0.216 & 0.309 & \multirow{2}{*}{ TBD } \\
\cline { 1 - 3 } Weight & 0.300 & 0.400 & 0.300 & \\
\hline
\end{tabular}

Table 2. The fuzzy distances of our algorithm and Lu Hui's research

\begin{tabular}{|l|c|c|c|}
\hline & \multicolumn{3}{|c|}{ Classic field } \\
\hline & Attribute 1 & Attribute 2 & Attribute 3 \\
\hline 1 & 0.215 & 0.059 & 0.162 \\
\hline 2 & 0.134 & 0.103 & 0.160 \\
\hline 3 & 0.106 & 0.033 & 0.068 \\
\hline
\end{tabular}

Table 3. The fuzzy closeness values of our algorithm and Lu Hui's research

\begin{tabular}{|l|c|c|c|c|}
\hline & \multicolumn{3}{|c|}{ Classic field } & \multirow{2}{*}{ Weighted fuzzy closeness } \\
\hline & Attribute 1 & Attribute 2 & Attribute 3 & \\
\hline 1 & 0.785 & 0.942 & 0.838 & 0.864 \\
\hline 2 & 0.866 & 0.897 & 0.840 & 0.870 \\
\hline 3 & 0.894 & 0.967 & 0.932 & 0.937 \\
\hline
\end{tabular}

Table 4. The initial data of our algorithm and Kemal Akyol's research

\begin{tabular}{|c|c|c|c|c|c|}
\hline & \multicolumn{4}{|c|}{ Classic field } & \multirow{2}{*}{ Target interval } \\
\hline & Attribute 1 & Attribute 2 & Attribute 3 & Attribute 4 & \\
\hline 1 & $0.069-0.158$ & $0.085-0.327$ & $0.146-0.196$ & $0.077-0.162$ & $0.080-0.180$ \\
\hline 2 & $0.083-0.181$ & $0.172-0.265$ & $0.127-0.275$ & $0.106-0.216$ & $0.180-0.280$ \\
\hline 3 & $0.187-0.327$ & $0.256-0.284$ & $0.292-0.332$ & $0.209-0.301$ & $0.280-0.400$ \\
\hline Predictive analysis target & 0.195 & 0.202 & 0.227 & 0.183 & \multirow{2}{*}{ TBD } \\
\hline Weight & 0.154 & 0.477 & 0.288 & 0.081 & \multirow{2}{*}{} \\
\hline
\end{tabular}

Table 5. The fuzzy distances of our algorithm and Kemal Akyol's research

\begin{tabular}{|l|c|c|c|c|}
\hline & \multicolumn{4}{|c|}{ Classic field } \\
\hline & Attribute 1 & Attribute 2 & Attribute 3 & Attribute 4 \\
\hline 1 & 0.093 & 0.121 & 0.061 & 0.076 \\
\hline 2 & 0.080 & 0.049 & 0.078 & 0.059 \\
\hline 3 & 0.094 & 0.069 & 0.087 & 0.085 \\
\hline
\end{tabular}

Table 6. The fuzzy closeness values of our algorithm and Kemal Akyol's research

\begin{tabular}{|c|c|c|c|c|c|}
\hline & \multicolumn{4}{|c|}{ Classic field } & \multirow{2}{*}{ Weighted fuzzy closeness } \\
\hline & Attribute 1 & Attribute 2 & Attribute 3 & Attribute 4 & 0.903 \\
\hline 1 & 0.907 & 0.879 & 0.939 & 0.924 & 0.939 \\
\hline 2 & 0.920 & 0.951 & 0.922 & 0.941 & 0.920 \\
\hline 3 & 0.906 & 0.931 & 0.913 & 0.915 & \\
\hline
\end{tabular}




\section{CONCLUSIONS}

The presence of fuzzy information has complicated the predictive analysis of complex systems under multiple constraints. For engineering applications, it is very meaningful to develop a predictive analysis method facing multiple fuzzy constraints. This paper puts forward an improved multiconstrained fuzzy predictive analysis algorithm. The author explained the normalization, weighting, granularity setting and classic domain of the attributes of multiple constraints, introduced the calculation of the fuzzy distance and fuzzy closeness for the attributes of multiple constraints, and detailed the realization of our algorithm and model multi-constrained fuzzy predictive analysis. The effectiveness and feasibility of our algorithm and model were demonstrated through comparison with relevant data in the literature. The results show that the results of our approach agree with those of the literature. The proposed algorithm and model provide a new solution and support to multi-constrained fuzzy predictive analysis.

\section{ACKNOWLEDGMENT}

1. Key scientific research projects of institutions of higher learning in Henan Province in 2020 (NO.20A520034): Research and development of task scheduling optimization in distributed system for resource sharing and load balancing.

2. Key R \& D and promotion special projects of Henan Province in 2020 (NO.202400450121): Research on the strategy of financial accounting system construction in Henan Province from the perspective of artificial intelligence.

3. Henan Province Teacher Education Curriculum Reform Project: Research on the curriculum reform of teachers' Informatization in primary and secondary schools under the mode of "Internet + education"(NO.2019-JSJYYB-118).

\section{REFERENCES}

[1] Zhang, L.M., Tang, W., Liu, Y.F., Lv, T. (2015). Multiobjective optimization and decision-making for DG planning considering benefits between distribution company and DGs owner. International Journal of Electrical Power \& Energy Systems, 73: 465-474. http://dx.doi.org/ 10.1016/j.ijepes.2015.05.019

[2] Guo, Q., Zou, G.T. (2017). Prediction method for extension architecture programming based on decision tree classification. CAAI Transactions on Intelligent Systems, 12(1): 117-123. http://dx.doi.org/10.11992/tis.201610015

[3] Majhi, B., Anish, C.M. (2015). Multiobjective optimization based adaptive models with fuzzy decision making for stock market forecasting. Neurocomputing, 502-511.

http://dx.doi.org/10.1016/j.neucom.2015.04.044

[4] Ekel, P., Kokshenev, I., Parreiras, R., Pedrycz, W., Pereira Jr, J. (2016). Multiobjective and multiattribute decision making in a fuzzy environment and their power engineering applications. Information Sciences, 361-362: 100-119. http://dx.doi.org/10.1016/j.ins.2016.04.030

[5] Lu, H. (2016). A grey forecasting model of the implicit correlation in complex system based on grey relation analysis method. Journal of Jiamusi University (Natural
Science $\quad$ Edition), 34(1): 110-113. http://dx.doi.org/10.3969/j.issn.1008-1402.2016.01.028

[6] Javed, S.A., Mahmoudi, A., Khan, A.M., Javed, S., Liu, S. (2018). A critical review: Shape optimization of welded plate heat exchangers based on grey correlation theory. Applied Thermal Engineering, 144: 593-599. https://doi.org/10.1016/j.applthermaleng.2018.08.086

[7] Bezuglov, A., Comert, G. (2016). Short-term freeway traffic parameter prediction: Application of grey system theory models. Expert Systems with Applications, 62: 284-292. http://dx.doi.org/10.1016/j.eswa.2016.06.032

[8] Memon, M.S., Lee, Y.H., Mari, S.I. (2015). Group multicriteria supplier selection using combined grey systems theory and uncertainty theory. Expert Systems with Applications, 42: 7951-7959. http://dx.doi.org/10.1016/j.eswa.2015.06.018

[9] Vukovic, D., Vyklyuk, Y., Chernova, N., Maiti, M. (2019). Neural network forecasting in prediction Sharpe ratio: Evidence from EU debt market. Physica A: Statistical Mechanics and its Applications, 123331. https://doi.org/10.1016/j.physa.2019.123331

[10] Navas, R.K.B., Prakash, S., Sasipraba, T. (2019). Artificial Neural Network based computing model for wind speed prediction: A case study of Coimbatore, Tamil Nadu, India. Physica A: Statistical Mechanics and its Applications, 123383. https://doi.org/10.1016/j.physa.2019.123383

[11] Akyol, K. (2020). Comparing of deep neural networks and extreme learning machines based on growing and pruning approach. Expert Systems with Applications, 140:

107-111. https://doi.org/10.1016/j.eswa.2019.112875

[12] Mao, Y., Wang, J., Lu, X., Mao, D., Gao, X. (2017). Research and application of uncertain genetic neural network in landslide hazard prediction. Computer Engineering, $\quad 43(2)$ : 308-316. https://doi.org/10.3969/j.issn.1000-3428.2017.02.052

[13] Wadkar, M., Di Troia, F., Stamp, M. (2020). Detecting malware evolution using support vector machines. Expert Systems with Applications, 2020: 143: 1-10. https://doi.org/10.1016/j.eswa.2019.113022

[14] Hou, Q., Liu, L., Zhen, L., Jing, L. (2018). A novel projection nonparallel support vector machine for pattern classification. Engineering Applications of Artificial Intelligence, 75: 64-75. https://doi.org/10.1016/j.engappai.2018.08.003

[15] Lukmanto, R.B., Nugroho, A., Akbar, H. (2019). Early detection of diabetes mellitus using feature selection and fuzzy support vector machine. Procedia Computer Science, 157: 46-54. https://doi.org/10.1016/j.procs.2019.08.140

[16] Gangsar, P., Tiwari, R. (2019). A support vector machine based fault diagnostics of Induction motors for practical situation of multi-sensor limited data case. Measurement, 135:

694-711. https://doi.org/10.1016/j.measurement.2018.12.011

[17] Lee, C.K.H. (2018). A review of applications of genetic algorithms in operations management. Engineering Applications of Artificial Intelligenc, 76: 1-12. https://doi.org/10.1016/j.engappai.2018.08.011

[18] Iyer, V.H., Mahesh, S., Malpani, R., Sapre, M., Kulkarni, A.J. (2019). Adaptive range genetic algorithm: A hybrid optimization approach and its application in the design and economic optimization of shell-and-tube heat 
exchanger. Engineering Applications of Artificial Intelligence, 85: 444-461. https://doi.org/10.1016/j.engappai.2019.07.001

[19] de Assis, L.S., Junior, J.R.D.P., Tarrataca, L., Haddad, D.B. (2019). Efficient Volterra systems identification using hierarchical genetic algorithms. Applied Soft Computing, 85 :

$1-12$. https://doi.org/10.1016/j.asoc.2019.105745

[20] Tseng, H.E., Chang, C.C., Lee, S.C., Huang, Y.M. (2018). A block-based genetic algorithm for disassembly sequence planning. Expert Systems with Applications, 96: 492-505. https://doi.org/10.1016/j.eswa.2017.11.004

[21] Lu, H. (2016). A multilevel extension association forecasting analysis model under the influence of many factors and its application of complex systems. Chong Qin Ke Ji Xue Yuan Xue Bao (Natural Science Edition), 18(3): 107-111. http://dx.doi.org/10.3969/j.issn.16731980.2016.03.030

[22] Chao, K.H. (2014). An extension theory-based maximum power tracker using a particle swarm optimization algorithm. Energy Conversion and Management, 86: 435-442. http://dx.doi.org/10.1016/j.enconman.2014.05.018

[23] Chao, K.H., Ching, J.L. (2010). An intelligent maximum power point tracking method based on extension theory for PV systems. Expert Systems with Applications, 37(2): 1050-1055.

http://dx.doi.org/10.1016/j.eswa.2009.06.068

[24] Silva, M.M., de Gusmão, A.P.H., Poleto, T., e Silva, L.C., Costa, A.P.C.S. (2014). A multidimensional approach to information security risk management using FMEA and fuzzy theory. International Journal of Information Management, 34(6): 733-740. http://dx.doi.org/10.1016/j.ijinfomgt.2014.07.005

[25] Magdalena, L. (2019). Semantic interpretability in hierarchical fuzzy systems: Creating semantically decouplable hierarchies. Information Sciences, 496: 109123. https://doi.org/10.1016/j.ins.2019.05.016

[26] Sakthivel, R., Raajananthini, K., Kwon, O.M., Mohanapriya, S. (2019). Estimation and disturbance rejection performance for fractional order fuzzy systems. ISA Transactions, 92: 65-74. https://doi.org/10.1016/j.isatra.2019.02.005 